\title{
Identification of dry and wet 6 months' period in eastern Slovakia using indices
}

\author{
Patrik Nagy ${ }^{1}$, Martina Zeleňáková ${ }^{*}$, Slávka Galas $^{2}$, Helena Hlavatá $^{3}$, Dorota Simonová ${ }^{3}$ \\ ${ }^{1}$ TUKE, Faculty of Civil Engineering, Department of Environmental Engineering, Vysokoskolska 4, \\ Kosice 042 00, Slovakia \\ ${ }^{2}$ AGH University of Science and Technology, Department of Environmental Analysis, Geological \\ Mapping and Economic Geology, al. Mickiewicza 30, 30-059 Krakow, Poland \\ ${ }^{3}$ Slovak Hydrometeorological Institute, Branch Office Košice, Košice 041 17, Slovakia
}

\begin{abstract}
In the paper we evaluated dry and wet 6 months' periods, which reflect changes in water resources of the country. We assessed Standardized Precipitation Index (SPI), Standardized Evapotranspiration Index (SPEI), Streamflow Drought Index (SDI), Reconnaissance Drought Index (RDI). The time period was 1960 - 2015 and the study area includes eastern Slovakia - selected water and climatic stations. The results indicate dry periods and wet periods. The results of work are presented in the table for separate evaluated indices.
\end{abstract}

\section{Introduction}

Earth's climate is changing. Temperatures are rising, snow and precipitation patterns are changing, and extreme climatic events - such as heavy rains and increasing temperatures are occurring. Climate change refers to major climate parameters (such as temperature or precipitation) that change during the longer periods. Natural factors have caused climate change during previous periods of Earth's history, but human activities are primarily due to the changes that are currently observed [1-3].

Extreme events such as floods and droughts are the most serious and the most natural risks associated with climate change. Drought is one of the most serious threats to people having adverse effects on the economy, industry [4]. Defining a drought is complicated depending on the area where it occurs, the length of time in which climate zone is perceived the observed area and other various aspects. The simplest definition of drought is a phenomenon that occurs during periods of subnormal precipitation from a duration of several days to several months [5]. Compared to the floods, drought slowly develops and is only acknowledged when changes in the environment begin to be felt and people begin to feel them. From this reason, it is difficult to determine when the drought started and when it ended. Initially, it occurs as subnormal precipitation and, for a long time, drought has an impact on the hydrological cycle. Evapotranspiration is mainly influenced by temperature. Temperature is one of the main factors affecting the availability of water and has a consequent effect on the occurrence of drought and the nature of drought [6-7].

\footnotetext{
*Corresponding author: martina.zelenakova@tuke.sk
} 
Drought indices are based on hydrological and meteorological parameters. The most commonly used indices droughts include the Palmer Index (PDSI) and the Standardized Precipitation Index (SPI) [8].

In this paper we evaluated the dry and wet periods by the drought indices: Standardized Precipitation Index (SPI), Standardized Evapotranspiration Index (SPEI), Reconnaissance Drought Index (RDI), Streamflow Drought Index (SDI). These indices differ in input parameters. The data were provided by the Slovak Hydrometeorological Institute, Košice Regional Office.

\section{Material and Methods}

As we mentioned, we have identified dry and wet seasons using SPI, SPEI, RDI and SDI. The evaluated indexes have different input parameters.

Negative values of indices mean dry period and positive values of indices mean wet period, while the intensity is graded in individual degrees.

Table 1. Classification value indices [2].

\begin{tabular}{|l|l|}
\hline Period & Value of index \\
\hline Extremely wet & 2.0 and more \\
\hline Very wet & 1.5 to 1.99 \\
\hline Moderately wet & 1 to 1.49 \\
\hline Near normal & -0.99 to 0.99 \\
\hline Moderately dry & -1 to -1.49 \\
\hline Severely dry & -1.5 to -1.99 \\
\hline Extremely dry & -2 and less \\
\hline
\end{tabular}

The Standardized Precipitation Index (SPI) uses precipitation records. SPIs can only be calculated on the basis of a minimum time series of 20 years, but ideally is, if the time series are 30 years old, even if data are missing. Time steps that can be evaluated are from 1 month to 48 months [7].

The Standardized Evapotranspiration Index (SPEI) uses the similar basis, but in addition to precipitation, it uses air temperature to calculate evapotranspiration [7].

The Streamflow Drought Index (SDI) uses input data of water discharge and SPI-related normalization methods [8].

The Reconnaissance Drought Index (RDI) contains a simplified water balance equation that includes precipitation and potential evapotranspiration. It has three outputs: initial value, normalized value and standardized value. We used a standardized value in this contribution. The standardized value of RDI is similar in nature to SPI and can be compared [9].

SPI, SDI and SPEI were calculated using Drin software. In addition, the software includes a module for estimating the potential evapotranspiration of potential evapotranspiration (PET) based on temperature for methods that are useful for calculating RDI. The software can be used in a variety of applications such as monitoring, spatial drought assessment, climatic and drought scenarios [8].

We calculated the SPEI using the statistical software RGui. It is a GNU project (Linux operating system) that is similar to the $\mathrm{S}$ language and environment. 


\section{Data and study area}

The water and climatological stations I evaluated are located in eastern Slovakia. The stations Poprad, Svit, Chmel'nica and Červený Kláštor are located in the Poprad and Dunajec basins on the Poprad River. The basin of Poprad and Dunajec is located in the north of eastern Slovakia. The stations Švedlár, Mníšek nad Hnilcom and Stratená are located in the catchment area Hornád on the river Hnilec. In the Bodrog basin there are stations Kamenica nad Cirochou, Humenne, Krasny Brod, Tisinec-Stropkov and Streda nad Bodrogom. Košice weather station is located in the Hornád basin. The latitude and longitude of the stations are shown in Table 2.

Table 2. Localization of assessment stations.

\begin{tabular}{|l|l|l|}
\hline Station & Latitude & Longitude \\
\hline Poprad & $49^{\circ} 3^{\prime} 41.18^{\prime \prime}$ & $20^{\circ} 17^{\prime} 52.73^{\prime \prime}$ \\
\hline Svit & $49^{\circ} 3^{\prime} 36.5^{\prime \prime}$ & $20^{\circ} 12^{\prime} 25.02^{\prime \prime}$ \\
\hline Chmel'nica & $49^{\circ} 17^{\prime} 60.0^{\prime \prime}$ & $20^{\circ} 43^{\prime} 59.99^{\prime \prime}$ \\
\hline Červený Kláštor & $49^{\circ} 23^{\prime} 30.48^{\prime \prime}$ & $20^{\circ} 24^{\prime} 19.08^{\prime \prime}$ \\
\hline Stratená & $48^{\circ} 52^{\prime} 12.72^{\prime \prime}$ & $20^{\circ} 22^{\prime} 38.64^{\prime \prime}$ \\
\hline Mníšek nad Hnilcom & $48^{\circ} 48^{\prime} 15.12^{\prime \prime}$ & $20^{\circ} 48^{\prime} 16.92^{\prime \prime}$ \\
\hline Švedlár & $48^{\circ} 48^{\prime} 59.99^{\prime \prime}$ & $20^{\circ} 42^{\prime} 59.99^{\prime \prime}$ \\
\hline Humenné & $48^{\circ} 56^{\prime} 13.45^{\prime \prime}$ & $21^{\circ} 54^{\prime} 58.5^{\prime \prime}$ \\
\hline Kamenica nad Cirochou & $48^{\circ} 55^{\prime} 59.99^{\prime \prime}$ & $22^{\circ} 00^{\prime} 0.00^{\prime \prime}$ \\
\hline Krásny Brod & $49^{\circ} 14^{\prime} 23^{\prime \prime}$ & $21^{\circ} 53^{\prime} 54^{\prime \prime}$ \\
\hline Tisinec-Stropkov & $49^{\circ} 12^{\prime} 7.6^{\prime \prime}$ & $21^{\circ} 39^{\prime} 7.78^{\prime \prime}$ \\
\hline Streda nad Bodrogom & $48^{\circ} 23^{\prime} 25.37^{\prime \prime}$ & $21^{\circ} 45^{\prime} 9.97^{\prime \prime}$ \\
\hline Košice & $48^{\circ} 42^{\prime} 50.22^{\prime \prime}$ & $21^{\circ} 15^{\prime} 29.09^{\prime \prime}$ \\
\hline
\end{tabular}

Table 3 shows the input values, the evaluation period and the average annual values of the indicators for the evaluation period from the data available to me. Data provided by SHMI were average monthly values of climatological and hydrological variables.

Table 3. Input data and evaluated period.

\begin{tabular}{|l|c|c|c|}
\hline \multicolumn{1}{|c|}{ Station } & $\begin{array}{c}\text { Input } \\
\text { variables }\end{array}$ & Assessed period & $\begin{array}{c}\text { Yearly average } \\
\text { values }\end{array}$ \\
\hline \multirow{2}{*}{ Poprad } & Precipitation & $1960-2014$ & $601,1[\mathrm{~mm}]$ \\
\cline { 2 - 4 } & Temperature & $1960-2014$ & $6,15\left[{ }^{\circ} \mathrm{C}\right]$ \\
\hline \multirow{2}{*}{ Svit } & Streamflow & $1965-2015$ & $1,29\left[\mathrm{~m}^{3} / \mathrm{s}\right]$ \\
\hline \multirow{2}{*}{ Chmel'nica } & Streamflow & $1960-2015$ & $15,1\left[\mathrm{~m}^{3} / \mathrm{s}\right]$ \\
\cline { 2 - 4 } & Precipitation & $1980-2010$ & $771[\mathrm{~mm}]$ \\
\hline Červený Kláštor & Temperature & $1961-2014$ & $6,34\left[{ }^{\circ} \mathrm{C}\right]$ \\
\hline Stratená & Streamflow & $1960-2015$ & $1,1\left[\mathrm{~m}^{3} / \mathrm{s}\right]$ \\
\hline Mníšek nad Hnilcom & Precipitation & $1980-2010$ & $710[\mathrm{~mm}]$ \\
\hline Švedlár & Temperature & $1961-2014$ & $6,56\left[{ }^{\circ} \mathrm{C}\right]$ \\
\hline Humenné & Streamflow & $1968-2015$ & $13,2\left[\mathrm{~m}^{3} / \mathrm{s}\right]$ \\
\hline
\end{tabular}




\begin{tabular}{|l|c|c|c|}
\hline & Precipitation & $1980-2014$ & $729,1[\mathrm{~mm}]$ \\
\hline Kamenica nad Cirochou & Temperature & $1960-2014$ & $8,7\left[{ }^{\circ} \mathrm{C}\right]$ \\
\hline \multirow{2}{*}{ Krásny Brod } & Streamflow & $1960-2015$ & $2,05\left[\mathrm{~m}^{3} / \mathrm{s}\right]$ \\
\cline { 2 - 4 } & Precipitation & $1986-2010$ & $831,7[\mathrm{~mm}]$ \\
\hline Tisinec-Stropkov & Temperature & $1963-2010$ & $8,14\left[{ }^{\circ} \mathrm{C}\right]$ \\
\hline \multirow{2}{*}{ Streda nad Bodrogom } & Precipitation & $1980-2010$ & $605,3[\mathrm{~mm}]$ \\
\cline { 2 - 4 } & Streamflow & $1960-2015$ & $110\left[\mathrm{~m}^{3} / \mathrm{s}\right]$ \\
\hline Košice & Temperature & $1960-2014$ & $9,1\left[{ }^{\circ} \mathrm{C}\right]$ \\
\hline
\end{tabular}

The input data, as was mentioned, were provided by the Slovak Hydrometeorological Institute.

\section{Results}

The results from the assessment is provided in the next Tables.

Two extremely dry seasons were recorded in Poprad and Svit in the observed time series during the years 1978-1979 and 13 very dry seasons in 1981-1982 were recorded as is seen from Table 4.

Table 4. Assessment of 6 monthly indices in Poprad/Svit drought seasons.

\begin{tabular}{|c|c|c|c|c|}
\hline \multicolumn{5}{|c|}{ Poprad/Svit } \\
\hline Indices & \multicolumn{2}{|c|}{ SDI } & \multicolumn{2}{|c|}{ SPI } \\
\hline Season & $\begin{array}{l}\text { October - } \\
\text { March }\end{array}$ & $\begin{array}{c}\text { April - } \\
\text { September }\end{array}$ & $\begin{array}{c}\text { October - } \\
\text { March }\end{array}$ & $\begin{array}{c}\text { April - } \\
\text { September }\end{array}$ \\
\hline $\begin{array}{c}\text { Extremely } \\
\text { dry }\end{array}$ & $\begin{array}{l}1978-1979 \\
1981-1982\end{array}$ & $\begin{array}{l}1978-1979 \\
1981-1982\end{array}$ & & \\
\hline $\begin{array}{c}\text { Severely } \\
\text { dry }\end{array}$ & $\begin{array}{l}1991-1992 \\
1982-1983\end{array}$ & $\begin{array}{l}1991-1992 \\
1982-1983\end{array}$ & 1981-1982 & \\
\hline Indices & \multicolumn{2}{|c|}{ RDI } & \multicolumn{2}{|c|}{ SPEI } \\
\hline $\begin{array}{c}\text { Extremely } \\
\text { dry }\end{array}$ & $\begin{array}{l}1973-1974 \\
1983-1984\end{array}$ & & & \\
\hline $\begin{array}{c}\text { Severely } \\
\text { dry }\end{array}$ & $\begin{array}{l}1971-1972 \\
1972-1973 \\
1977-1978 \\
2001-2002\end{array}$ & $\begin{array}{l}\text { 1981-1982 } \\
1985-1986\end{array}$ & $1986-1987$ & $\begin{array}{l}\mathbf{1 9 8 1}-\mathbf{1 9 8 2} \\
2002-2003 \\
2011-2012\end{array}$ \\
\hline
\end{tabular}

Periods that are bold are repeated in multiple indexes.

In Table 5, extremely humid and very humid periods are evaluated. There were 5 extremely wet seasons and 11 very wet seasons.

Table 5. Assessments of 6 monthly indices in Poprad/Svit wet seasons.

\begin{tabular}{|c|c|c|c|c|}
\hline \multicolumn{4}{|c|}{ Poprad/Svit } \\
\hline Indices & \multicolumn{2}{|c|}{ SDI } & \multicolumn{2}{c|}{ SPI } \\
\hline Season & $\begin{array}{c}\text { October - } \\
\text { March }\end{array}$ & $\begin{array}{c}\text { April - } \\
\text { September }\end{array}$ & $\begin{array}{c}\text { October - } \\
\text { March }\end{array}$ & $\begin{array}{c}\text { April - } \\
\text { September }\end{array}$ \\
\hline
\end{tabular}




\begin{tabular}{|c|c|c|c|c|}
\hline $\begin{array}{c}\text { Extremely } \\
\text { wet }\end{array}$ & 2000-2001 & $2000-2001$ & 1974-1975 & 1974-1975 \\
\hline $\begin{array}{c}\text { Severely } \\
\text { wet }\end{array}$ & $\begin{array}{l}1971-1972 \\
1999-2000\end{array}$ & $\begin{array}{r}1971-1972 \\
1999-2000\end{array}$ & $\begin{array}{l}1964-1965 \\
1976-1977 \\
2012-2013\end{array}$ & $\begin{array}{l}1962-1963 \\
1964-1965\end{array}$ \\
\hline Indices & \multicolumn{2}{|c|}{ RDI } & \multicolumn{2}{|c|}{ SPEI } \\
\hline $\begin{array}{c}\text { Extremely } \\
\text { wet }\end{array}$ & 1974-1975 & $\begin{array}{c}1971-1972 \\
\mathbf{2 0 0 9 - 2 0 1 0}\end{array}$ & 1974-1975 & \\
\hline $\begin{array}{c}\text { Severely } \\
\text { wet }\end{array}$ & $\begin{array}{r}1962-1963 \\
1964-1965\end{array}$ & $\begin{array}{l}2000-2001 \\
2013-2014\end{array}$ & 2010-2011 & $\begin{array}{l}1969-1970 \\
\mathbf{1 9 7 1 - 1 9 7 2} \\
\mathbf{2 0 0 9 - 2 0 1 0}\end{array}$ \\
\hline
\end{tabular}

There were 5 extreme drought periods observed at the stations Streda nad Bodrogom and Kosice, as is seen in Table 6.

Table 6. Assessments of 6 monthly indices in Streda nad Bodrogom/Košice dry seasons.

\begin{tabular}{|c|c|c|c|c|}
\hline \multicolumn{5}{|c|}{ Streda nad Bodrogom/Košice } \\
\hline Indices & \multicolumn{2}{|c|}{ SDI } & \multicolumn{2}{|c|}{ SPI } \\
\hline Season & $\begin{array}{c}\text { October - } \\
\text { March }\end{array}$ & $\begin{array}{c}\text { April - } \\
\text { September }\end{array}$ & $\begin{array}{l}\text { October - } \\
\text { March }\end{array}$ & $\begin{array}{c}\text { April - } \\
\text { September }\end{array}$ \\
\hline $\begin{array}{c}\text { Extremely } \\
\text { dry }\end{array}$ & $1963-1964$ & $1960-1961$ & & \\
\hline $\begin{array}{c}\text { Severely } \\
\text { dry }\end{array}$ & $\begin{array}{l}1972-1973 \\
1989-1990\end{array}$ & $2006-2007$ & $\begin{array}{l}1972-1973 \\
1976-1977 \\
1983-1984\end{array}$ & \\
\hline Indices & \multicolumn{2}{|c|}{ RDI } & \multicolumn{2}{|c|}{ SPEI } \\
\hline $\begin{array}{c}\text { Extremely } \\
\text { dry }\end{array}$ & $1986-1987$ & $\begin{array}{c}1991-1992 \\
\mathbf{2 0 0 2}-\mathbf{2 0 0 3} \\
\end{array}$ & & \\
\hline $\begin{array}{c}\text { Severely } \\
\text { dry }\end{array}$ & $\begin{array}{l}1996-1997 \\
1997-1998\end{array}$ & $\begin{array}{l}1992-1993 \\
1993-1994 \\
2001-2002 \\
2008-2009\end{array}$ & & $2002-2003$ \\
\hline
\end{tabular}

In bold, the periods that have been repeated are highlighted.

Seven extremely humid seasons and seven very humid seasons were occurring in Streda nad Bodrogom and Košice, see Table 7.

Table 7. Assessmnets of 6 monthly indices in Streda nad Bodrogom/Košice wet seasons.

\begin{tabular}{|c|c|c|c|c|}
\hline \multicolumn{5}{|c|}{ Streda nad Bodrogom/Košice } \\
\hline Indices & \multicolumn{2}{|c|}{ SDI } & \multicolumn{2}{c|}{ SPI } \\
\hline Season & $\begin{array}{c}\text { October - } \\
\text { March }\end{array}$ & $\begin{array}{c}\text { April - } \\
\text { September }\end{array}$ & $\begin{array}{c}\text { October - } \\
\text { March }\end{array}$ & $\begin{array}{c}\text { April - } \\
\text { September }\end{array}$ \\
\hline $\begin{array}{c}\text { Extremely } \\
\text { wet }\end{array}$ & $1974-1975$ & $\begin{array}{c}1979-1980 \\
2009-2010\end{array}$ & $1990-1991$ & $1990-1991$ \\
\hline $\begin{array}{c}\text { Severely } \\
\text { wet }\end{array}$ & $1966-1967$ & $1984-1985$ & & \\
\hline
\end{tabular}




\begin{tabular}{|c|c|c|c|c|}
\hline & \multicolumn{2}{|c|}{$2005-2006$} & & \\
\hline Indices & \multicolumn{2}{|c|}{ RDI } & & $\mathbf{2 0 0 9 - 2 0 1 0}$ \\
\hline $\begin{array}{c}\text { Extremely } \\
\text { wet }\end{array}$ & $\begin{array}{c}1981-1982 \\
1999-2000\end{array}$ & & & SPEI \\
\hline $\begin{array}{c}\text { Severely } \\
\text { wet }\end{array}$ & $\mathbf{1 9 9 8 - 1 9 9 9}$ & & $\mathbf{1 9 7 9 - 1 9 8 0}$ & $\mathbf{1 9 7 9 - 1 9 8 0}$ \\
\hline
\end{tabular}

One extremely dry period was recorded in Humenné and Kamenica nad Cirochou, and only in the SDI index. Very dry periods occurred in seven cases, see Table 8.

Table 8. Assessments of 6 monthly indices in Humenné/Kamenica nad Cirochou dry seasons.

\begin{tabular}{|c|c|c|c|c|}
\hline \multicolumn{5}{|c|}{ Humenné/Kamenica nad Cirochou } \\
\hline Indices & \multicolumn{2}{|c|}{ SDI } & \multicolumn{2}{|c|}{ SPI } \\
\hline Season & $\begin{array}{l}\text { October - } \\
\text { March }\end{array}$ & $\begin{array}{c}\text { April - } \\
\text { September }\end{array}$ & $\begin{array}{c}\text { October - } \\
\text { March } \\
\end{array}$ & $\begin{array}{c}\text { April - } \\
\text { September }\end{array}$ \\
\hline $\begin{array}{c}\text { Extremely } \\
\text { dry }\end{array}$ & 1976-1977 & 1976-1977 & & \\
\hline $\begin{array}{c}\text { Severely } \\
\text { dry }\end{array}$ & $\begin{array}{l}1988-1989 \\
2006-2007\end{array}$ & $\begin{array}{l}1988-1989 \\
2006-2007\end{array}$ & $\begin{array}{l}1972-1973 \\
1973-1974 \\
1982-1983 \\
\end{array}$ & \\
\hline $\begin{array}{c}\text { Indices } \\
\text { Extremely } \\
\text { dry }\end{array}$ & & & & \\
\hline $\begin{array}{c}\text { Severely } \\
\text { dry }\end{array}$ & 1984-1985 & $\begin{array}{l}2002-2003 \\
2006-2007\end{array}$ & 1984-1985 & $\begin{array}{l}2002-2003 \\
2006-2007\end{array}$ \\
\hline
\end{tabular}

Two extremely wet seasons were recorded in Humenné and Kamenica nad Cirochou, 1990-1991 in the SPI index and 2009-2010 in the SPEI index. Very wet periods occurred in the SDI, RDI and SPEI index, see Table 9.

Table 9. Assessments of 6 monthly indices in Humenné/Kamenica nad Cirochou wet seasons.

\begin{tabular}{|c|c|c|c|c|}
\hline \multicolumn{5}{|c|}{ Humenné/Kamenica nad Cirochou } \\
\hline Indices & \multicolumn{2}{|c|}{ SDI } & \multicolumn{2}{|c|}{ SPI } \\
\hline Season & $\begin{array}{c}\text { October - } \\
\text { March }\end{array}$ & $\begin{array}{c}\text { April - } \\
\text { September }\end{array}$ & $\begin{array}{c}\text { October - } \\
\text { March }\end{array}$ & $\begin{array}{c}\text { April - } \\
\text { September }\end{array}$ \\
\hline $\begin{array}{c}\text { Extremely } \\
\text { wet }\end{array}$ & & & 1990-1991 & 1990-1991 \\
\hline $\begin{array}{c}\text { Severely } \\
\text { wet }\end{array}$ & $\begin{array}{l}1967-1968 \\
1969-1970 \\
1991-1992\end{array}$ & $\begin{array}{l}1967-1968 \\
1969-1970 \\
1991-1992\end{array}$ & & \\
\hline Indices & \multicolumn{2}{|c|}{ RDI } & \multicolumn{2}{|c|}{ SPEI } \\
\hline $\begin{array}{l}\text { Extremely } \\
\text { wet }\end{array}$ & & & & 2009-2010 \\
\hline $\begin{array}{c}\text { Severely } \\
\text { wet }\end{array}$ & $\begin{array}{c}1980-1981 \\
1985-1986 \\
1998-1999 \\
1999-2000\end{array}$ & & $\begin{array}{l}2002-2003 \\
2008-2009 \\
\mathbf{2 0 0 9}-2010\end{array}$ & \\
\hline
\end{tabular}


Four extremely dry periods in SDI and RDI were recorded in Krasny Brod and Tisinec. Very dry periods were recorded for ten cases and occurred in all observation indices, see Table 10 .

Table 10. Assessments of 6 monthly indices in Krásny Brod/Tisinec dry seasons.

\begin{tabular}{|c|c|c|c|c|}
\hline \multicolumn{5}{|c|}{ Krásny Brod/Tisinec } \\
\hline Indices & \multicolumn{2}{|c|}{ SDI } & \multicolumn{2}{|c|}{ SPI } \\
\hline Season & $\begin{array}{c}\text { October - } \\
\text { March }\end{array}$ & $\begin{array}{c}\text { April - } \\
\text { September }\end{array}$ & $\begin{array}{c}\text { October - } \\
\text { March }\end{array}$ & $\begin{array}{c}\text { April - } \\
\text { September }\end{array}$ \\
\hline $\begin{array}{c}\text { Extremely } \\
\text { dry }\end{array}$ & 1983-1984 & & & \\
\hline $\begin{array}{c}\text { Severely } \\
\text { dry }\end{array}$ & $\begin{array}{l}1962-1963 \\
1995-1996\end{array}$ & $\begin{array}{l}2006-2007 \\
2014-2015\end{array}$ & $\begin{array}{c}1963-1964 \\
1969-1970\end{array}$ & $\begin{array}{l}1963-1964 \\
1969-1970\end{array}$ \\
\hline Indices & \multicolumn{2}{|c|}{ RDI } & \multicolumn{2}{|c|}{ SPEI } \\
\hline $\begin{array}{c}\text { Extremely } \\
\text { dry }\end{array}$ & & $\begin{array}{l}1993-1994 \\
1999-2000 \\
2002-2003\end{array}$ & & \\
\hline $\begin{array}{c}\text { Severely } \\
\text { dry }\end{array}$ & & $\begin{array}{c}1987-1988 \\
1990-1991 \\
1994-1995 \\
1998-1999\end{array}$ & & 1988-1989 \\
\hline
\end{tabular}

Four extremely wet seasons were recorded in Krásny Brod and Tisinec in the SDI and RDI indices. Very dry periods were recorded in ten and occurred in SDI, SPI, RDI and SPEI, see Table 11.

Table 11. Assessments of 6 monthly indices in Krásny Brod/Tisinec wet seasons.

\begin{tabular}{|c|c|c|c|c|}
\hline \multicolumn{5}{|c|}{ Krásny Brod/Tisinec } \\
\hline Indices & \multicolumn{2}{|c|}{ SDI } & \multicolumn{2}{|c|}{ SPI } \\
\hline Season & $\begin{array}{l}\text { October - } \\
\text { March }\end{array}$ & $\begin{array}{c}\text { April - } \\
\text { September }\end{array}$ & $\begin{array}{l}\text { October - } \\
\text { March }\end{array}$ & $\begin{array}{c}\text { April - } \\
\text { September }\end{array}$ \\
\hline $\begin{array}{c}\text { Extremely } \\
\text { wet }\end{array}$ & & $\begin{array}{l}1979-1980 \\
\text { 2009-2010 }\end{array}$ & & \\
\hline $\begin{array}{c}\text { Severely } \\
\text { wet }\end{array}$ & $\begin{array}{l}1978-1979 \\
1999-2000\end{array}$ & 2004-2005 & 1984-1985 & 1984-1985 \\
\hline Indices & \multicolumn{2}{|c|}{ RDI } & \multicolumn{2}{|c|}{ SPEI } \\
\hline $\begin{array}{c}\text { Extremely } \\
\text { wet }\end{array}$ & $\begin{array}{l}1991-1992 \\
1994-1995 \\
\end{array}$ & & & \\
\hline $\begin{array}{c}\text { Severely } \\
\text { wet }\end{array}$ & $\begin{array}{l}1986-1987 \\
1987-1988 \\
1992-1993 \\
1999-2000\end{array}$ & 2009-2010 & 2009-2010 & $1990-1991$ \\
\hline
\end{tabular}

Periods that are highlighted in bold appear multiple times.

Three extremely dry periods in the SDI and RDI index were found in Chmel'nica and Červený Kláštor. Very dry periods were found in ten cases see Table 12. 
Table 12. Assessments of 6 monthly indices in Chmel’nica/Červený Kláštor dry seasons.

\begin{tabular}{|c|c|c|c|c|}
\hline \multicolumn{5}{|c|}{ Chmel'nica/Červený kláštor } \\
\hline Indices & \multicolumn{2}{|c|}{ SDI } & \multicolumn{2}{|c|}{ SPI } \\
\hline Season & $\begin{array}{c}\text { October - } \\
\text { March }\end{array}$ & $\begin{array}{c}\text { April - } \\
\text { September }\end{array}$ & $\begin{array}{c}\text { October - } \\
\text { March }\end{array}$ & $\begin{array}{c}\text { April - } \\
\text { September }\end{array}$ \\
\hline $\begin{array}{c}\text { Extremely } \\
\text { dry }\end{array}$ & 1977-1978 & 1977-1978 & & \\
\hline $\begin{array}{c}\text { Severely } \\
\text { dry }\end{array}$ & $\begin{array}{l}1967-1968 \\
2005-2006\end{array}$ & $\begin{array}{l}1967-1968 \\
2005-2006\end{array}$ & $\begin{array}{l}\text { 1960-1961 } \\
1962-1963\end{array}$ & 1960-1961 \\
\hline Indices & \multicolumn{2}{|c|}{ RDI } & \multicolumn{2}{|c|}{ SPEI } \\
\hline $\begin{array}{c}\text { Extremely } \\
\text { dry }\end{array}$ & & $\begin{array}{l}1980-1981 \\
1986-1987\end{array}$ & & \\
\hline $\begin{array}{c}\text { Severely } \\
\text { dry }\end{array}$ & & $\begin{array}{c}1987-1988 \\
1992-1993 \\
1993-1994 \\
2002-2003\end{array}$ & & $\begin{array}{l}1979-1980 \\
1984-1985\end{array}$ \\
\hline
\end{tabular}

In Chmel'nica and Červený Kláštor were recorded two extremely wet seasons. The period 1993-1994 occurred in the SDI index and the period 1999-2000 occurred in the RDI index. In the SPI index, an extremely wet and very wet period was not found. Three very wet periods were recorded in the SDI index, five very wet periods occurred in the RDI index, and two wet periods were recorded in the SPEI index, see Table 13.

Table 13. Assessments of 6 monthly indices in Chmel'nica/Červený Kláštor wet seasons.

\begin{tabular}{|c|c|c|c|c|}
\hline \multicolumn{5}{|c|}{ Chmel'nica/Červený kláštor } \\
\hline Indices & \multicolumn{2}{|c|}{ SDI } & \multicolumn{2}{|c|}{ SPI } \\
\hline Season & $\begin{array}{c}\text { October - } \\
\text { March }\end{array}$ & $\begin{array}{c}\text { April - } \\
\text { September }\end{array}$ & $\begin{array}{c}\text { October - } \\
\text { March }\end{array}$ & $\begin{array}{c}\text { April - } \\
\text { September }\end{array}$ \\
\hline $\begin{array}{c}\text { Extremely } \\
\text { wet }\end{array}$ & 1993-1994 & 1993-1994 & & \\
\hline $\begin{array}{c}\text { Severely } \\
\text { wet }\end{array}$ & $\begin{array}{l}1960-1961 \\
1968-1969 \\
1970-1971\end{array}$ & $\begin{array}{c}1960-1961 \\
1968-1969 \\
1970-1971\end{array}$ & & \\
\hline Indices & \multicolumn{2}{|c|}{$\begin{array}{l}\text { RDI } \\
\end{array}$} & \multicolumn{2}{|c|}{ SPEI } \\
\hline $\begin{array}{c}\text { Extremely } \\
\text { wet }\end{array}$ & $1999-2000$ & & & \\
\hline $\begin{array}{c}\text { Severely } \\
\text { wet }\end{array}$ & $\begin{array}{l}1982-1983 \\
1985-1986 \\
1991-1992 \\
1994-1995 \\
2009-2010\end{array}$ & & & $\begin{array}{l}1999-2000 \\
2002-2003\end{array}$ \\
\hline
\end{tabular}

At the evaluated stations Stratená, Švedlár and Mníšek nad Hnilcom, one extremely dry period was found in the SDI index and three extremely dry periods were recorded in the RDI index. Very dry periods occurred in eight cases, see Table 14. 
Table 14. Assessments 6 monthly indices Stratená/Švedlár/Mníšek nad Hnilcom dry season.

\begin{tabular}{|c|c|c|c|c|}
\hline \multicolumn{5}{|c|}{ Stratená/Švedlár/Mníšek nad Hnilcom } \\
\hline Indices & \multicolumn{2}{|c|}{ SDI } & \multicolumn{2}{|c|}{ SPI } \\
\hline Season & $\begin{array}{c}\text { October - } \\
\text { March }\end{array}$ & $\begin{array}{c}\text { April - } \\
\text { September }\end{array}$ & $\begin{array}{c}\text { October - } \\
\text { March }\end{array}$ & $\begin{array}{c}\text { April - } \\
\text { September }\end{array}$ \\
\hline $\begin{array}{c}\text { Extremely } \\
\text { dry }\end{array}$ & & $1992-1993$ & & \\
\hline $\begin{array}{c}\text { Severely } \\
\text { dry }\end{array}$ & \begin{tabular}{|l|l|}
$1983-1984$ \\
$1986-1987$ \\
\end{tabular} & $\begin{array}{l}1960-1961 \\
\mathbf{2 0 0 2}-\mathbf{2 0 0 3} \\
\end{array}$ & $\begin{array}{l}\mathbf{1 9 8 1}-1982 \\
1982-1983 \\
\end{array}$ & \\
\hline Indices & \multicolumn{2}{|c|}{ RDI } & \multicolumn{2}{|c|}{ SPEI } \\
\hline $\begin{array}{c}\text { Extremely } \\
\text { dry }\end{array}$ & \begin{tabular}{|l|}
$\mathbf{1 9 8 3 - 1 9 8 4}$ \\
$2001-2002$ \\
\end{tabular} & $1985-1986$ & & \\
\hline $\begin{array}{c}\text { Severely } \\
\text { dry }\end{array}$ & $1989-1990$ & $\begin{array}{l}1980-1981 \\
1981-1982 \\
1986-1987 \\
1992-1993 \\
2002-2003 \\
\end{array}$ & & 2002-2003 \\
\hline
\end{tabular}

Eight extreme wet seasons and eight very wet seasons were recorded in Stratená, Švedlár and Mníšek nad Hnilcom in SDI, SPI and RDI, see Table 15.

Table 15. Assessments of 6 monthly indices in Stratená/Švedlár/Mníšek nad Hnilcom wet seasons.

\begin{tabular}{|c|c|c|c|c|}
\hline \multicolumn{5}{|c|}{ Stratená/Švedlár/Mníšek nad Hnilcom } \\
\hline Indices & \multicolumn{2}{|c|}{ SDI } & \multicolumn{2}{|c|}{ SPI } \\
\hline Season & $\begin{array}{l}\text { October - } \\
\text { March }\end{array}$ & $\begin{array}{c}\text { April - } \\
\text { September }\end{array}$ & $\begin{array}{c}\text { October - } \\
\text { March }\end{array}$ & $\begin{array}{c}\text { April - } \\
\text { September }\end{array}$ \\
\hline $\begin{array}{c}\text { Extremely } \\
\text { wet }\end{array}$ & $\begin{array}{l}1974-1975 \\
1976-1977 \\
2010-2011\end{array}$ & $\begin{array}{l}\mathbf{1 9 6 4 - 1 9 6 5} \\
1971-1972\end{array}$ & $1989-1990$ & \\
\hline $\begin{array}{c}\text { Severely } \\
\text { wet }\end{array}$ & $\begin{array}{l}\text { 1964-1965 } \\
1980-1981\end{array}$ & $\begin{array}{l}\text { 1977-1978 } \\
\mathbf{2 0 0 9 - 2 0 1 0} \\
2012-2013\end{array}$ & 1985-1986 & $1985-1986$ \\
\hline Indices & \multicolumn{2}{|c|}{ RDI } & \multicolumn{2}{|c|}{ SPEI } \\
\hline $\begin{array}{l}\text { Extremely } \\
\text { wet }\end{array}$ & $\begin{array}{l}1982-1983 \\
\mathbf{2 0 0 9}-2010\end{array}$ & 2009-2010 & 2009-2010 & 2009-2010 \\
\hline $\begin{array}{c}\text { Severely } \\
\text { wet }\end{array}$ & $\begin{array}{l}1998-1999 \\
2008-2009\end{array}$ & & & \\
\hline
\end{tabular}

Periods that are highlighted in a bold font colour appear in multiple indexes.

\section{Conclusions}

The contribution was focused on the evaluation of dry and wet periods using SDI, SPI, RDI and SPEI drought indices in time series 1960-2015 in a time step of 6 months. Time step 6 months reflects changes in water accumulation in water reservoirs. The results of the evaluated sites show alternation of dry and wet periods. Extremely wet seasons and very dry seasons that occurred most frequently at the monitored stations were 1960-1961, 1963- 
1964, 1967-1968, 1969-1970, 1976-1977, 1977-1978, 1978-1979, 1981-1982, 1983- 1984, 1984-1985, 1986-1987, 1988-1989, 1992-1993, 2002-2003,2005-2006, 2006-2007. Extremely wet seasons and the most wet seasons were: 1960-1961, 1962-1963, 1964-1965, 1968-1969, 1970-1971, 1971-1972, 1974-1975, 1979-1980, 1984-1985, 1985-1986, 19901991, 1993-1994, 1998-1999, 1999-2000, 2000-2001, 2009-2010. As can be seen in the hydrological years 1960-1961 and 1984-1985, the 6-month wet period alternated with the dry 6-month period.

As a result, it is possible to propose adaptation measures for better water management in the evaluated areas. Adaptation measures may be an overestimation of water levels in river basins in the dry season and water retention in the wet season.

Thanks to the support of project SK-PL-18-0033 by Slovak Research and Development Agency.

\section{References}

1. K. Mishra, V. P. Singh, J Hydrol 391, 202-216 (2010)

2. M. Fendeková, J. Poórová, V. Slivová, Hydrologické sucho na Slovensku a prognóza jeho vývoja (Bratislava: Comenius University, 2018)

3. Didovets, V. Krysanova, G. Bürger, S. Snizhko, V. Balabukh, A. Bronstert, J Hydrol, 22, (2019)

4. M. Milanovic, M. Gocic, S. Trajkovic, Agriculture and agricultural science procedia, 4, 167-174 (2015)

5. H. West, Q. Nevil, H. Michael, Remote Sens Environ 232 (2019)

6. S. Tirivarombo, D. Osupile, P. Eliasson, Phys Chem Earth, Parts A/B/C 106, 1-10 (2018)

7. M. Svoboda, M. Hayes, D. Wood, Standardized precipitation index user guide (World Meteorological Organization Geneva, Switzerland 2012)

8. D. Tigkas, H. Vangelis, G. Tsakiris, Earth Sci Inform, 8, 697-709 (2015)

9. M. Svoboda, B. Fuchs, Handbook of drought indicators and indices (World Meteorological Organization and Global Water Partnership 2016)

10. SHMI, Climate Atlas of Slovakia (Bratislava: Slovenský hydrometeorologický ústav, 2015) 